\title{
La Ley de Migración y su Armonización con la Ley General de Acceso de las Mujeres a una Vida Libre de Violencia en el Caso de las Niñas y las Adolescentes Migrantes
}

\author{
Gabriela Ivette Herrera Valdez ${ }^{1}$ \\ Maria Auxiliadora Moreno Valenzuela ${ }^{2}$ \\ Jesús Omar Pérez Luna ${ }^{3}$ \\ Universidad de Sonora
}

Sumario: I. Introducción. II. Género en la Migración. III. Causas del Aumento de la Migración de Las Niñas Niños y Adolescentes. IV. El Marco Normativo Migratorio y su armonización con la Ley General de Acceso de las Mujeres a una Vida Libre de Violencia. V. Conclusiones.

\section{Resumen}

En este trabajo se analizó la Ley General de Migración para identificar si se encuentra armonizada con la Ley general de Acceso de las Mujeres a una Vida Libre de Violencia y sí en consecuencia establece acciones diferenciadas para la protección y atención de las niñas y mujeres migrantes víctimas de delitos en y durante el tránsito migratorio.

Palabras claves: Armonización, género, derechos humanos, niñas y mujeres adolescentes.

\begin{abstract}
On this paper I analized The General Law of Migration to identify if it is harmonized with the General Law of Acces for Women to a Life Out of Violence and if, on consequence, it states diferential actions for the protection and atention of the migrants girls and women victims of felanies in and during the migrant transit.
\end{abstract}

Key words: harmonization, gender, human rights, girls and women, teenagers.

\footnotetext{
${ }^{1}$ Estudiante de la Licenciatura en derecho de la Universidad de Sonora

2 Docente Investigadora del Departamento de Derecho integrante del CAEC 141 de la Universidad de Sonora

${ }^{3}$ Estudiante de la Licenciatura en derecho de la Universidad de Sonora
} 


\section{Introducción.}

Amnistía Internacional reporta que el $80 \%$ de las mujeres y niñas migrantes que provienen de Centroamérica, son violadas mientras cruzan por México. Igual sucede con otros delitos como el de trata de personas, en donde ellas aparecen como las principales víctimas. Estudios de género señalan que las oportunidades, experiencias y exclusiones para las niñas y niños inmigrantes están diferenciadas en función del género, así resulta pertinente analizar, cómo lo hacemos en este documento, la forma en que las niñas y mujeres adolescentes migrantes viven los riesgos del tránsito migratorio, en relación a los niños y hombres adolescentes. Efectivamente tanto hombres como mujeres, viven la violencia del tránsito desde su género, razón que explica como ellas tratan de migrar acompañadas por un hombre con el fin de disminuir la posibilidad de ser atacada por otros hombres durante su camino "hacia el norte", mientras que se ha documentado cómo ellos tratan de proteger a las mujeres que viajan con ellos o a las que encuentran durante el tránsito migratorio. La revisión de si la Ley de Migración se encuentra armonizada con la Ley General de Acceso de las Mujeres a una Vida Libre de Violencia nos permitió la reflexión sobre la inclusión de la perspectiva de género en dicha normativa a fin de ampliar la protección específica de las mujeres y de las niñas y adolescentes migrantes en México.

\section{Género en la migración.}

Género es el término que socialmente se utiliza para poder identificar las diferencias entre los sexos, que han sido construidas en la historia de relaciones sociales y se transforman constantemente a través de la cultura, así que no ésta, no es una palabra que se utiliza únicamente para designar 
la situación social de las mujeres. Cuando hablamos de sexo nos referimos exclusivamente a las similitudes biológicas entre hombres y mujeres. ${ }^{4}$

En la sociedad históricamente se han venido construyendo roles determinados por el género, las mujeres desde su niñez se les instruye para atender las labores domésticas, crianza, cuidado y educación de los hijos "propias de las mujeres" y los hombres desde su niñez también se les imponen roles representativos de los hombres como el "ser jefes de familia", proveedores responsables de sostener económicamente el hogar.

En el contexto social, dentro del cual ocurre la migración se reproducen igualmente los roles y estereotipos de género, tanto en el ámbito social como en las relaciones familiares, incluso cuando hablamos de personas en situaciones tan complejas como haber sido víctimas de delito, encontramos que las mujeres son más propensas a ser víctimas de delitos sexuales en relación a los hombres. Esto sucede de igual forma cuando hablamos de niñas niños y adolescentes, como los veremos más adelante en el desarrollo del presente trabajo.

De manera generalizada, las mujeres migrantes están más expuestas, a la explotación sexual, la trata, la prostitución forzada y a otras formas de violencia; así como a condiciones de trabajo precarias, menores salarios y mayores riesgos para su salud, entre ellos, el contacto con pesticidas.

\footnotetext{
"Organización Internacional para las Migrantes, "Género y Migración", disponible en http://www.mujeresenred.net/spip.php?article1519. P.5 -7.
} 
En los primeros estudios sobre migración a las mujeres migrantes se les ha venido reconociendo como mujeres dependientes, es decir que la decisión de migrar dependía directamente de su cónyuge o pareja, como parte de un proceso de reunificación familiar, donde los esposos eran quienes viajaban primero y después se trasladaban las mujeres y familiares. Sin embargo el incremento de las causas del desplazamiento forzado no solo aumentó la migración masculina, sino género además, las primeras generaciones de mujeres migrantes las cuales se vieran obligadas a insertarse en el proceso migratorio sea para poder contraer matrimonio o para trabajar en maquiladoras o servicios domésticos, lo que posibilitó que posteriormente la decisión de migrar no fuera dependiente de una solicitud o requerimiento lo que permitió además que poco a poco se les reconociera como agentes independientes y jefas de familia o estrategas. ${ }^{5}$

Podemos decir que el género como herramienta de análisis en la migración es importante en tanto que, permite analizar en forma diferenciada las razones por las que los hombres y las mujeres migran y como lo hacen, así como viven de igual forma los riesgos del tránsito migratorio. Es decir, los contextos y las experiencias les impactan en forma distinta y mucho más si hablamos de las niñas y los niños y las y los adolescentes.

En la actualidad, las mujeres dan razón de casi la mitad de la población migrante en todo el mundo, son más numerosas que los varones migrantes en los países desarrollados. A escala mundial: se contemplan en un $49.6 \%$ así lo señala en el informe anual del UNFPA. ${ }^{6}$

\footnotetext{
5 Mujeres en Red, "Las Mujeres y la Migración Internacional", El periódico Feminista: fecha de consulta el día 14 de junio de 2015. Disponible en http://www.mujeresenred.net/IMG/article_PDF/article_a800.pdf

${ }^{6}$ UNFPA estado de la población mundial (2006). Hacia la esperanza: las mujeres y la mujer, internacional. Disponible en http://www.unfpa.org/sites/default/files/pub-pdf/sowp06-sp.pdf. P. 1
} 
En México, la Secretaría de Gobernación (Segob) alerta sobre la creciente presencia de niñas y adolescentes mujeres en los flujos migratorios hacia Estados Unidos, tanto Centroamericanas como Mexicanas. Estadísticas de la patrulla fronteriza Estadounidense, indican que "de octubre de 2013 hasta junio de 2014, más de 51,000 menores de edad han sido detenidos por la Patrulla Fronteriza"7

El documento elaborado por la Unidad de Política Migratoria de la Secretaría de Gobernación señala que, "entre 2010 y 2013, las mujeres representan entre 25 y 27 por ciento de los menores migrantes detenidos por el Instituto Nacional de Migración (INM), mientras que entre enero y septiembre de 2014 la presencia femenina entre los menores asegurados llegó a 36.2 por ciento".

En el año 2014, se observó un incremento significativo de la migración de niñas, niños y adolescentes centroamericanos hacia Estados Unidos, "6 mil 263 niñas y adolescentes centroamericanas fueron presentadas ante el INM, 135 por ciento más que en 2013".

III. Causas del aumento de la migración de las niñas, niños y adolescentes. Las condiciones que propician la migración de las niñas, niños y adolescentes migrantes se deben a un conjunto de factores que se viven en sus lugares de origen. La migración se convierte en una salida o escape de las violencias en sus países de origen, que puede ser ejercidas por grupos

\footnotetext{
${ }^{7}$ Estadísticas de la patrulla fronteriza estadounidense. U.S. Border Patrol Statistics. Fecha de consulta el día 23 de julio de 2015. Disponible en http://insyde.org.mx/wpcontent/uploads/2014/07/Fact-sheet-Ni\%C3\%B1ez-migrante-no-acompa\%C3\%B1ada-en-laregi\%C3\%B3n-norte-y-centroam\%C3\%A9rica.pdf

${ }^{8}$ Instituto para las Mujeres en la Migración (2013). México y las mujeres migrantes. Fecha de consulta el día 30 de julio de 2015. Disponible en

http://www.imumi.org/index.php?option=com_content\&view=article\&id=16\&ltemid=117
} 
delictivos armados desde los carteles del narcotráfico, las guerrillas, las pandillas, hasta las violencias institucionalizadas, sumado a esto la violencia intrafamiliar, y violencia sexual que se vive en algunos casos, tolerado por la persistencia de la impunidad y la incapacidad de los gobiernos para procurar la protección de sus nacionales.

El deseo de reunirse con sus familiares, la posibilidad de mejorar su nivel de vida a través del desempeño de un trabajo bien remunerado, son variables que se conjuntan con la posibilidad de huir de la pobreza, segregación, falta de oportunidades de educación y los estereotipos de género que se dan sobre todo en sectores rurales e indígenas, condiciones todas que se viven en sus lugares de origen y que se entrelazan para obligar a las niñas, niños $y$ adolescentes -que en muchas ocasiones quedan al frente del cuidado del hogar y la familia- a tomar la decisión de emprender el viaje. ${ }^{9}$

IV.- Los riesgos en la migración se viven de manera diferente respecto al Género.

Las mujeres y las adolescentes antes de ingresar al territorio mexicano, para no salir embarazadas se protegen con inyecciones anticonceptivas porque tienen la certeza que pueden ser abusadas sexualmente en el camino. Ciertamente se ha documentado cómo las mujeres, las niñas y las adolescentes son víctimas de violencia sexual cuando caen en manos del crimen organizado, los tratantes de blancas y los coyotes. Incluso han sido atacadas por sus mismos compañeros de viaje y hasta por servidores públicos y policías, para abusarlas y/o explotarlas sexualmente.

\footnotetext{
${ }^{9}$ Anaiz Zamora Márquez (2015) Cimacnoticias. Fecha de consulta el día 23 de junio de 2015. Disponible en http://www.cimacnoticias.com.mx/node/64176
} 
El abuso y violencia sexual es de los principales riesgos que existe en el camino ya que es el precio exigible a pagar por las mujeres migrantes para poder continuar el trayecto; otra manera es la negociación, que se da cuando una mujer pacta ser pareja sexual de un hombre, éste a cambio le ofrece protección en el trayecto, lo más difícil, sin duda es cuando se presenta el abuso producto de un secuestro ${ }^{10}$, ya que son sometidas a golpizas pero "Ahí no sólo reciben golpizas y amenazas sino que ellas son violadas una y otra vez; entonces esta cosificación del cuerpo femenino se resalta en todo el camino de tránsito", así expresó.

Amnistía internacional señala que un $80 \%$ de las niñas y las adolescentes mujeres son abusadas sexualmente antes de que puedan llegar a la frontera con Estados Unidos, viviendo un mayor riesgo cuando viajan sin documentos y por eso mismo, por áreas aisladas, evitando a las autoridades o arriba de los techos de trenes, lo que las convierte en presa fácil de las bandas de delincuencia organizada que operan en esos espacios territoriales. ${ }^{11}$

Las niñas y las adolescentes viven un proceso migratorio que se puede describir como una pesadilla sin limitaciones con un contenido de abusos y violencias. Además mediante estos abusos y violencias se reproducen los estereotipos de género ya que durante el viaje al ser cooptadas por las organizaciones criminales son forzadas a realizar trabajos domésticos de asistencia a los trabajadores de estas bandas delictivas, como lavarles sus ropas, preparar sus comidas mantener las áreas de trabajo limpias y por la

\footnotetext{
${ }^{10}$ Hiroko Asakura, (S.f). Seminario Migrantes entre Fronteras: Escenarios del Siglo 21", en Colegio de la Frontera Norte.

${ }^{11}$ Revista Animal Político. Fecha de consulta el día 28 de mayo de 2015. Disponible en http://www.animalpolitico.com/2014/09/80-de-mujeres-y-ninas-migrantes-centroamericanasson-violadas-en-mexico-al-intentar-cruzar-eu/
} 
noche servir de damas de compañía y en ocasiones pueden llegar a recibir "un sueldo" realmente simbólico, a diferencia de los niños y los adolescentes, que son cooptados para la realización de trabajos contemplados como actividades realizadas únicamente por los hombres y para las cuales estas bandas criminales los enseñan y les dan responsabilidades en los "negocios" delictivos tales como pasar personas o grupos de personas a Estados Unidos. Un ejemplo de esta situación son los "los coyotitos", que son niños que van desde edades de 11 a 17 años que se dedican a "pasar" personas sin documentos, en los diversos puertos fronterizos. Muchos de estos niños son cooptados por otros niños a los cuales les pagan para engancharlos y son "convencidos" explicándoles que no los pueden meter a la cárcel si los detienen, y que si no hablan pueden ganar hasta 100 dólares por persona. Kevin uno de los niños que fue entrevistado por la revista Diez 4 , afirma haber trasladado desde Tijuana a San Diego a 200 personas en dos años. ${ }^{12}$ Otros "negocios" en los que son integrados son formar parte de las filas de gatilleros en los grupos del narcotráfico o bien son utilizados como distribuidores de drogas, ya que siendo niños es más fácil pasar desapercibidos.

La violencia y el abuso diferenciado persigue a las niñas y niños y adolescentes desde sus lugares de origen, durante el tránsito y las y los alcanza, hasta el lugar de "las oportunidades" en Estados Unidos, donde realizan trabajos que son menos remunerados, labores domésticas donde las niñas y adolescentes son violentadas por sus "patronas" y violadas por sus "patrones" o son explotadas como sexoservidoras. Mientras que los

\footnotetext{
${ }^{12}$ Revista Diez de periodismo narrativo contra toda circunstancia. Fecha de consulta el día 1 de junio de 2015. Disponible en http://diez4.com/2012/los-coyotitos-no-van-a-parar/
} 
niños y los adolescentes son llevados a los grandes campos agricultores obligándolos a trabajo forzado bajo altas temperaturas y con sueldos muy poco remunerados.

V. El marco normativo migratorio y su armonización con la Ley General de Acceso de las Mujeres a una Vida Libre de Violencia.

En este apartado revisamos si la Ley de Migración se encuentra armonizada con la Ley General de Acceso de las Mujeres a una Vida Libre de Violencia con respecto a la protección de las mujeres migrantes contra la violencia de género, nos interesó específicamente identificar si la Ley de Migración incorpora acciones diferenciadas para proteger a las niñas y las adolescentes que transitan por el territorio mexicano rumbo a los Estados Unidos de Norteamérica.

Los Derechos Humanos de las niñas, niños y adolescentes migrantes se encuentran contemplados en la Ley de migración y su Reglamento, en la Circular por la que se instruye el procedimiento para la atención de los niños, niñas y adolescentes migrantes no acompañados y en la Circular por la que instruye el procedimiento que deberá seguir el Instituto Nacional de Migración en la detección, identificación y atención de personas extranjeras víctimas del delito, entre otros.

De igual forma las niñas, niños y adolescentes migrantes, son titulares de los derechos y garantías ${ }^{13}$ reconocidas a los niños en general, tanto en la

\footnotetext{
13 Para algunas corrientes del derecho con enfoque positivista todos aquellos derechos que no cuenten con las correspondientes garantías para que se de su cumplimiento son simplemente declamaciones retóricas o vagos programas políticos jurídicamente irrelevantes, por lo tanto no son derechos. Ferrajoli clasifica las garantías en primarias y secundarias. Por garantías primarias explica que se tratan de las obligaciones 0 prohibiciones correlativas a los derechos y por garantías secundarias se entiende a las obligaciones de aplicar sanciones o declaraciones de nulidad de las violaciones de las garantías primarias.
} 
Constitución Política de los Estados Unidos Mexicanos, la Ley general de los derechos de las niñas niños y adolescentes, y además en el ámbito internacional, la Convención sobre los Derechos de la Niñez, la Convención sobre la Eliminación de Todos las Formas de Discriminación contra la Mujer (CEDAW) y todos los demás instrumentos internacionales. En la fracción VI del artículo 50 de la citada Ley General de Acceso, se estipula, que se entenderá por víctima, a la mujer de cualquier edad a quien se le inflige cualquier tipo de violencia. Es decir la Ley acoge en este concepto a las niñas y las mujeres adolescentes a quien se les provoca algún tipo de violencia. ${ }^{14}$

De igual forma, la Ley en comento, señala cinco tipos de violencia: la violencia psicológica, la física, la patrimonial y la violencia económica y sexual. Las mujeres y las niñas y adolescentes migrantes que recorren el país rumbo a los Estados Unidos de Norteamérica de manera irregular, son víctimas de todas las modalidades de violencia que señala la Ley de Acceso.

"Muchas de las mujeres y niñas en tránsito por México, salen huyendo de un ambiente de violencia en sus lugares de origen, sin embargo uno de los problemas es que, en su huida, la violencia las encuentra de frente. La violencia está presente prácticamente a lo largo de todo el camino del tránsito migratorio. Las modalidades de la violencia que con más frecuencia enfrentan las niñas y las adolescentes migrantes, es aquella que ocurre en y durante el tránsito, dado a la falta de documentos se desplazan por lugares

\footnotetext{
${ }^{14}$ Ley General de Acceso de las Mujeres a una Vida Libre de Violencia. Publicada en el Diario Oficial de la Federación (D.O.F) Última reforma publicada 15-01-2013.
} 
poco transitados entre los montes y durante la noche, por lo que el riesgo aumenta." ${ }^{\prime 15}$

La Ley General de Acceso de las Mujeres a una Vida Libre de Violencia no establece una protección diferenciada para las niñas y las adolescentes por su minoría de edad esto tiene una consecuencia práctica ya que las características de la atención que requiere una mujer adulta con respecto a una niña y una adolescente son diferente o tendrían que serlo. Ya que cuentan con tres características que los y las hace diferentes en cuanto a los adultos la primera de ellas es su desarrollo cognitivo la niña o el niño se concentran en su propio punto de vista, lo que le dificulta considerar el de otra persona. Una niña o un niño no pueden acceder al razonamiento abstracto y desde el razonamiento concreto dan respuestas "incoherentes" para la lógica adulta.

"En el desarrollo psicológico de las personas de menor edad, las emociones inundan la realidad del niño o niña y la aparición de mecanismos de defensa inconscientes modifican la conducta y el pensamiento infantil para minimizar la angustia sin que el niño o niña pueda tener control sobre ellos." ${ }^{\text {16 }}$

Otra de las tres características tiene que ver con el desarrollo moral del niño, durante cierta etapa de su desarrollo priorizan por encima de cualquier otro factor la necesidad de evitar el castigo (evitar el castigo es lo correcto), de modo que al sentirse en riesgo de un castigo buscará dar la respuesta

\footnotetext{
${ }^{15}$ Instituto para las Mujeres en la Migración (2013). Fecha de consulta el día 25 de julio de 2015. Disponible en http://www.imumi.org/index.php?option=com_content\&view=article\&id=16\&ltemid=117 16 Suprema Corte de justicia de la Nación (2012). Protocolo de Actuación de Justicia en casos que afecten a niñas, niños y adolescentes. Fecha de consulta el día 29 de junio de 2015. Disponible en http://www.derechorevistajuridica.uson.mx/revistas/articulos/11-art5.pdf
} 
correcta considerará que desea la persona adulta por encima de lo que percibió a través de sus propios sentidos con relación a un hecho. ${ }^{17}$

En este aspecto de reconocimiento de derechos diferenciados, la ley de migración va más allá de la Ley General de Acceso al reconocer a las mujeres a las niñas, niños y adolescentes como grupos situación de vulnerabilidad $^{18}$, incluso hace un reconocimiento de la situación de vulnerabilidad que deviene de la irregularidad está contemplada en la ley donde indica que las personas migrantes (incluyendo por supuesto a las personas de menor edad) serán titulares de todos los derechos contemplados en ella, en su Reglamento. Sin embargo, y a pesar de este reconocimiento, la ley de migración se ve limitada al establecer los mecanismos de atención y protección específicos para estos sectores, esto es evidente en todo el texto de la Ley pero especialmente en el capítulo VII en donde se contempla el Procedimiento para la atención de personas en situación de vulnerabilidad" en donde a pesar del titulo se enfoca principalmente en las niñas, niños y adolescentes migrantes no acompañados (el viajar solos constituye una agravación más de la situación de vulnerabilidad que les deviene a éstos por su minoría de edad) pero sin

${ }^{17}$ Suprema Corte de justicia de la Nación (2012). Protocolo de Actuación para quienes Imparten Justicia en caso de que afecten a Niñas, Niños y Adolescentes. Fecha de consulta el día 2 de julio de 2015. Disponible en http://www.pjetam.gob.mx/tamaulipas/interiores/Publicaciones/Protocolo2012_v3.pdf

18 Artículo 73. La Secretaría deberá implementar acciones que permitan brindar una atención adecuada a los migrantes que por diferentes factores o la combinación de ellos, enfrentan situaciones de vulnerabilidad como son las niñas, niños y adolescentes migrantes no acompañados, las mujeres, las víctimas de delitos, las personas con discapacidad y las adultas mayores. 
hacer la distinción diferenciada, en la atención y protección que deberán recibir las niñas y las adolecentes en relación a los niños y los adolescentes.

\section{Conclusiones}

El Poder Legislativo al momento de elaborar la Ley de Migración omitió hacerla compatible con los derechos reconocidos a las mujeres en el ámbito internacional como en -el ámbito nacional- con los derechos y garantías establecidas para ellas en la Ley General de Acceso a una Vida Libre de Violencia. Tarea que es obligada si queremos evitar conflictos entre leyes y otorgar eficacia total a los derechos y garantías contenidas en la Ley de Migración, a favor de las mujeres migrantes y particularmente de las niñas y las adolescentes que transitan por nuestro país.

La colaboración de funcionarios públicos como policías y militares con las bandas dedicadas al secuestro y trata de personas, delitos de los cuales las personas migrantes son las principales víctimas, posibilita la corrupción y la consecuente impunidad.

El escepticismo que las migrantes experimentan frente a la temática de los Derechos Humanos. Esta postura se crea a partir de un incumplimiento, por parte del Estado como responsable de velar por las garantías individuales y los derechos de la población, la reiterada inobservancia y la frecuente impunidad estatal de esta responsabilidad constitucional fomentando la falta de confianza en las autoridades, las instituciones que son las que hacen posible el cumplimiento de la garantía que nos otorgan los derechos contemplados en las normas jurídicas, porque las mujeres que han sido víctimas de alguna forma de reproducción de violencia, son inhibidas se 


\section{abstienen de presentar una denuncia formal, para una niña o una adolescente esto es meramente poco reproducible.}

\section{Bibliografía y Web grafía}

Anaiz Zamora Márquez (2015) Cimacnoticias. Fecha de consulta el día 23 de junio de 2015 .

Disponible en http://www.cimacnoticias.com.mx/node/64176

Estadísticas de la patrulla fronteriza estadounidense. U.S. Border Patrol Statistics. Fecha de consulta el día 23 de julio de 2015. Disponible en http://insyde.org.mx/wpcontent/uploads/2014/07/Fact-sheet-Ni\%C3\%B1ez-migrante-no-acompa\%C3\%B1ada-en-laregi\%C3\%B3n-norte-y-centroam\%C3\%A9rica.pdf

Hiroko Asakura, (S.f). Seminario Migrantes entre Fronteras: Escenarios del Siglo $21 "$, en Colegio de la Frontera Norte.

Instituto para las Mujeres en la Migración (2013). México y las mujeres migrantes. Fecha de consulta el día 30 de julio de 2015. Disponible en http://www.imumi.org/index.php?option=com_content\&view=article\&id=16\&ltemid=117

Ley General de Acceso de las Mujeres a una Vida Libre de Violencia. Publicada en el Diario Oficial de la Federación (D.O.F) Última reforma publicada 15-01-2013.

Mujeres en Red, "Las Mujeres y la Migración Internacional", El periódico Feminista: fecha de consulta el día 14 de junio de 2015. Disponible en http://www.mujeresenred.net/IMG/article_PDF/article_a800.pdf

Organización Internacional para las Migrantes, "Género y Migración”, disponible en http://www.mujeresenred.net/spip.php?article1519. P.5 -7.

Revista Animal Político. Fecha de consulta el día 28 de mayo de 2015. Disponible en: http://www.animalpolitico.com/2014/09/80-de-mujeres-y-ninas-migrantescentroamericanas-son-violadas-en-mexico-al-intentar-cruzar-eu/

Revista Diez de periodismo narrativo contra toda circunstancia. Fecha de consulta el día 1 de junio de 2015. Disponible en http://diez4.com/2012/los-coyotitos-no-van-a-parar/

Suprema Corte de justicia de la Nación (2012). Protocolo de Actuación de Justicia en casos que afecten a niñas, niños y adolescentes. Fecha de consulta el día 29 de junio de 2015. Disponible en http://www.derechorevistajuridica.uson.mx/revistas/articulos/11-art5.pdf

Suprema Corte de justicia de la Nación (2012). Protocolo de Actuación para quienes Imparten Justicia en caso de que afecten a Niñas, Niños y Adolescentes. Fecha de consulta el día 2 de julio de 2015. Disponible en http://www.pjetam.gob.mx/tamaulipas/interiores/Publicaciones/Protocolo2012_v3.pdf

UNFPA estado de la población mundial (2006). Hacia la esperanza: las mujeres y la mujer, internacional. Disponible en http://www.unfpa.org/sites/default/files/pub-pdf/sowp06sp.pdf.P. 1 